\title{
Helicity amplitudes and crossing relations for antiproton proton reactions
}

\author{
N. H. Buttimore and E. Jennings* \\ School of Mathematics, Trinity College, Dublin, Ireland
}

February 2, 2008

\begin{abstract}
Antiproton proton annihilation reactions allow unique access to the moduli and phases of nucleon electromagnetic form factors in the time like region. We present the helicity amplitudes for the unequal mass single photon reaction $p \bar{p} \rightarrow l^{+} l^{-}$in the $s$ channel including the lepton mass. The relative signs of these amplitudes are determined using simple invariance properties. Helicity amplitudes for the annihilation reaction $p \bar{p} \rightarrow N \bar{N}$ are also given, where $N$ is any spinor particle with structure. Crossing relations between the $e p \rightarrow e p$ scattering and the $p \bar{p} \rightarrow l^{+} l^{-}$ annihilation channels are discussed and the crossing matrix for the helicity amplitudes is given. This matrix may be used to verify known expressions for the space like helicity amplitudes due to one photon exchange.
\end{abstract}

\section{Introduction}

Electromagnetic form factors contain information on the internal dynamics and structure of the nucleon. Form factors provide a description of baryons as composites and are related to the Fourier transforms of charge and magnetisation density distributions of the nucleon. These fundamental functions of momentum transfer indicate the hadron's internal structure, allowing us to probe the quark core as well as providing information on the parton model.

At present there is considerable interest in this area mainly due to the surprising result that, for example, the proton time like form factor is found to be twice as large as in the space like region [1] and that evaluations of the electromagnetic form factor ratio $G_{E} / G_{M}$ show a deviation from Rosenbluth scaling. Recent measurements of the electric proton form factor at large values of momentum transfer squared, $q^{2}$, show discrepancies in the results depending on whether a recoil polarisation method was used or the spin averaged differential cross section in electron proton

*elise@maths.tcd.ie 
scattering $[2,3]$. It has been suggested that these results may be resolved if we consider the two photon exchange mechanism taking place in the reaction as well as performing more experiments in the time like region in order to access the phases of these form factors. Recent discussions on this subject $[4,5]$ as well as extraction of proton form factors in the time like region [6] have reviewed and highlighted the need for further study and experimentation in this area.

The Polarised Antiproton Experiment (PAX) collaboration [7] aims to shed new light on nucleon electromagnetic form factors by accessing single and double spin observables. They plan to do this by using polarised antiprotons, produced by spin filtering with an internal polarised gas target. It is through these experiments that they hope to measure the moduli and the relative phase of the time like electric and magnetic form factors of the proton. The extensive spin programme at RHIC (BNL) also aims to provide new information on nucleon electromagnetic form factors $[8,9]$.

While there are several papers which discuss and present equations for the moduli and phases of the proton form factors in the time like region, [10], [11] and [5], there are advantages to considering helicity transition amplitudes for two body annhilation reactions [12]. Helicity amplitudes are particularly useful in analysing a scattering process as conservation of angular momentum in the centre of mass frame and spin dependent observables can be expressed simply. Also symmetry principles such as parity conservation and time reversal invariance can be stated directly in terms of helicity amplitudes which allows us to easily determine the number of independent amplitudes for a given process.

It is well known that in the space like regime the Dirac and Pauli form factors are real; however in the time like region they are complex on the real axis above the threshold $4 m_{\pi}^{2}$, where $m_{\pi}$ is the pion mass. These two regimes are connected by dispersion relations which permit analytic continuation of the amplitudes from the time like to the space like region. It is these analytic properties which give rise to a crossing matrix [13] between the helicity amplitudes for the $t$ channel reaction $l^{-} p \rightarrow l^{-} p$ and the $s$ channel reaction $p \bar{p} \rightarrow l^{+} l^{-}$. This crossing matrix is presented in section 5 .

The paper is organised as follows: in section 2 we outline the calculation and results for the five independent helicity amplitudes for the $s$ channel reaction $p \bar{p} \rightarrow$ $l^{+} l^{-}$. It is shown that the squared moduli of the amplitudes sum to the spin averaged differential cross section for the same process, confirming that helicity amplitudes contribute incoherently to the unpolarised cross section. Section 3 employs the symmetry properties of charge conjugation, parity and time reversal invariance in order to determine the relative signs of these amplitudes.

In section 4 we present the helicity amplitudes for the annihilation reaction $N \bar{N} \rightarrow p \bar{p}$ in terms of four form factors. The final section reviews the crossing relations between the scattering, $t$, and the annihilation, $s$, channels and discusses the Wigner rotations needed to transform from one channel to another. We give the crossing matrix $W$ which connects the proton lepton scattering helicity amplitudes to the amplitudes for proton antiproton annihilation giving rise to a lepton antilepton pair. This matrix, $W$, is a $(6 \times 6)$ matrix whose entries depend on the Mandelstam variables $s, t$ and on $m$ and $M$ the masses of the lepton and proton 
respectively. The crossing matrix $W$ reduces to a previously published result when $m=M[14]$.

\section{$2 \quad$ Helicity amplitudes for $l^{-} l^{+} \rightarrow \bar{p} p$}

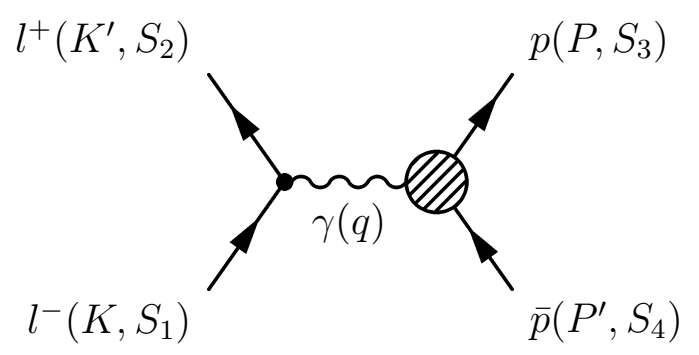

Figure 1: One photon exchange for $l^{-}+l^{+} \rightarrow \bar{p}+p$ in the $s$ channel.

In calculating the helicity amplitudes for lepton antilepton annihilation leading to a proton antiproton pair, figure 1, or the time reversed reaction we can firstly find the differential cross sections for the reaction involving pure helicity states. Taking the square root of this cross section yields the absolute value of the helicity amplitude. We discuss the relative signs of these amplitudes in section 3. The formulae used for the differential cross section in terms of the invariant amplitude $\mathcal{M}$ is given in appendix $\mathrm{A}$.

When all four spinors relate to polarised fermions the invariant amplitude for lepton antilepton to proton antiproton due to one photon exchange is

$\mathcal{M}=i e^{2} \bar{v}\left(K^{\prime}, S_{2}\right) \gamma_{\mu} u\left(K, S_{1}\right) \frac{1}{s} \bar{u}\left(P, S_{3}\right)\left[\gamma^{\mu} F_{1}(s)+\frac{i}{2 M} \sigma^{\mu \nu} q_{\nu} F_{2}(s)\right] v\left(P^{\prime}, S_{4}\right)$

where $M$ is the mass of the proton and $\sigma^{\mu \nu}=\frac{1}{2} i\left[\gamma^{\mu}, \gamma^{\nu}\right]$. The four momenta of the incoming lepton and antilepton are $K^{\mu}$ and $K^{\prime \mu}$ respectively and $P^{\nu}$ and $P^{\prime \nu}$ are the four momenta of the outgoing proton and antiproton. These momenta as well as the spin four vectors, $S_{i}, i \in\{1,2,3,4\}$, are given in the centre of mass frame for the time reversed reaction in appendix B. Here $s=q^{2}=q_{\mu} q^{\mu}$ is the square of the invariant 4 -momentum in the s channel. So that $q^{2}$ is positive in the time like region we adopt the metric $q^{2}=q_{0}^{2}-\mathbf{q}^{2}$. This is given in terms of the final proton and antiproton momenta by

$$
q^{\nu}=P^{\nu}+P^{\prime \nu}
$$

The Dirac and Pauli form factors, $F_{1}$ and $F_{2}$, are analytic functions of $q^{2}$. They take real values in the space like region $q^{2}<0$ due to the hermiticity of the Hamiltonian. The form factors are complex in the time like region on the real $q^{2}$ axis above 
threshold. Using Gordon decomposition this amplitude becomes

$$
\begin{aligned}
\mathcal{M}=i e^{2} \bar{v} & \left(K^{\prime}, S_{2}\right) \gamma_{\mu} u\left(K, S_{1}\right) \frac{1}{s} \\
& \times \bar{u}\left(P, S_{3}\right)\left[\gamma^{\mu} G_{M}(s)+\frac{1}{2 M}\left(P^{\prime}-P\right)^{\mu} F_{2}(s)\right] v\left(P^{\prime}, S_{4}\right) .
\end{aligned}
$$

The Sachs electric and magnetic form factors $G_{E}$ and $G_{M}$ are a linear combination of $F_{1}$ and $F_{2}$ and are defined in appendix A.

Using the Mathematica package "Tracer.m" we can evaluate the proton and lepton current. The proton current requires us to evaluate the following trace:

$$
\operatorname{Tr}(\not P+M)\left(1+\gamma_{5} \mathscr{S} / 3\right)\left[\gamma^{\nu} G_{M}+R^{\nu} F_{2}\right]\left(\not \not^{\prime}-M\right)\left(1+\gamma_{5} \mathscr{S} 4\right)\left[\gamma^{\mu} G_{M}^{*}+R^{\mu} F_{2}^{*}\right]
$$

where $R_{\mu}=\left(P_{\mu}{ }^{\prime}-P_{\mu}\right) / 2 M$ and $*$ denotes complex conjugation. Here as we are not summing over proton polarisations we have $u\left(P, S_{3}\right) \bar{u}\left(P, S_{3}\right)=(\not P-M) / 2$. The lepton current involves a slightly simpler trace,

$$
\operatorname{Tr}(K+m)\left(1+\gamma_{5} \mathscr{S}_{1}\right) \gamma_{\mu}\left(K^{\prime}-m\right)\left(1+\gamma_{5} \mathscr{S}_{2}\right) \gamma_{\nu}
$$

where $m$ denotes the lepton mass. Once each of these traces is calculated and then contracted together we arrive at the differential cross section for the process.

We shall briefly outline the notation used in the following sections. The helicity amplitudes describing the $s$ channel reaction

$$
l^{-}+l^{+} \rightarrow \bar{p}+p
$$

are written as

$$
H\left(\lambda_{\bar{p}} \lambda_{p} ; \lambda_{l^{-}} \lambda_{l^{+}}\right)
$$

where $\lambda_{i}$ is the helicity of particle $i$, which is either + or - depending on which helicity amplitude we are considering. We find the following five independent cross sections for this process:

$$
\begin{aligned}
& \frac{d \sigma}{d \Omega}(++;++)=\frac{d \sigma}{d \Omega}(++;--)=\frac{d \sigma}{d \Omega}(--;--)=\frac{d \sigma}{d \Omega}(--;++) \\
& \frac{d \sigma}{d \Omega}(++;+-)=\frac{d \sigma}{d \Omega}(++;-+)=\frac{d \sigma}{d \Omega}(--;-+)=\frac{d \sigma}{d \Omega}(--;+-) \\
& \frac{d \sigma}{d \Omega}(+-;--)=\frac{d \sigma}{d \Omega}(+-;++)=\frac{d \sigma}{d \Omega}(-+;--)=\frac{d \sigma}{d \Omega}(-+;++) \\
& \frac{d \sigma}{d \Omega}(+-;+-)=\frac{d \sigma}{d \Omega}(-+;-+) \\
& \frac{d \sigma}{d \Omega}(+-;-+)=\frac{d \sigma}{d \Omega}(-+;+-) .
\end{aligned}
$$

Taking the square root of the cross section gives us the absolute value of the single photon helicity amplitude in each case. The expression for the centre of mass 
scattering angle, $\theta$, depends on both the lepton and proton mass and is given in appendix B.

$$
\begin{aligned}
\frac{1}{\alpha}|H(++;++)| & =\frac{2 m M}{s} \cos \theta\left|G_{E}\right| \\
\frac{1}{\alpha}|H(+-;+-)| & =\frac{1}{2}(1+\cos \theta)\left|G_{M}\right| \\
\frac{1}{\alpha}|H(+-;-+)| & =\frac{1}{2}(1-\cos \theta)\left|G_{M}\right| \\
\frac{1}{\alpha}|H(++;+-)| & =\frac{M}{\sqrt{s}} \sin \theta\left|G_{E}\right| \\
\frac{1}{\alpha}|H(+-;--)| & =\frac{m}{\sqrt{s}} \sin \theta\left|G_{M}\right|
\end{aligned}
$$

In the above equation we can see that the helicity amplitude $H(+-;+-)$ vanishes at $\theta=180^{\circ}$. This is what we would expect since for $\theta=180^{\circ}$ the total angular momentum of the final state is opposite to that of the initial state.

We may check that the squares of the helicity amplitudes, Eq. (2.9), sum to the spin averaged differential cross section for this reaction. The spin averaged differential cross section for $l^{-} l^{+} \rightarrow \bar{p} p$ is [5]

$$
\begin{aligned}
\frac{d \sigma}{d \Omega}=\alpha^{2} \beta & \frac{1}{s^{3}\left(s-4 M^{2}\right)}\left\{\frac{s^{2}}{2}\left(s-4 M^{2}\right)\left|G_{M}\right|^{2}-4 s m^{2} M^{2}\left(\left|G_{M}\right|^{2}-\left|G_{E}\right|^{2}\right)\right. \\
& \left.+\left[\left(t-m^{2}-M^{2}\right)^{2}+s t\right]\left(s\left|G_{M}\right|^{2}-4 M^{2}\left|G_{E}\right|^{2}\right)\right\} .
\end{aligned}
$$

The variable $\beta$ is a flux factor defined in appendix $\mathrm{A}$. The spin averaged cross section can also be written in terms of the centre of mass scattering angle, $\theta$, as

$$
\begin{aligned}
\frac{4 s}{\alpha^{2} \beta} \frac{d \sigma}{d \Omega}=\left(1+\cos ^{2} \theta\right)\left|G_{M}\right|^{2}+\frac{4 M^{2}}{s} \sin ^{2} \theta\left|G_{E}\right|^{2} \\
\\
\quad \frac{4 m^{2}}{s} \sin ^{2} \theta\left|G_{M}\right|^{2}+\frac{16 m^{2} M^{2}}{s^{2}} \cos ^{2} \theta\left|G_{E}\right|^{2} .
\end{aligned}
$$

This unpolarised cross section for spin $1 / 2$ particles is related to the sum of the helicity amplitudes listed above as

$$
s \frac{d \sigma}{d \Omega}=\frac{1}{4} \sum_{\lambda}\left|H\left(\lambda_{\bar{p}} \lambda_{p} ; \lambda_{l^{-}} \lambda_{l^{+}}\right)\right|^{2} .
$$

The sum in the above equation is over all sixteen helicity amplitudes. Time reversal invariance reduces this number to eight; using parity invariance lowers this again to six. As the reaction involves identical particles charge conjugation invariance may be used to reduce the number of independent helicity amplitudes to five. 
Using the five independent amplitudes that we found in Eq. (2.9) above this becomes

$$
\begin{aligned}
s \frac{d \sigma}{d \Omega}_{\text {unpol }}=\mid H( & ++;+)\left.\right|^{2}+|H(++;+-)|^{2}+|H(+-;--)|^{2} \\
& +\frac{1}{2}|H(+-;+-)|^{2}+\frac{1}{2}|H(+-;-+)|^{2} .
\end{aligned}
$$

Looking at the formula for the spin averaged cross section given in Eq. (2.11) we can see it is the sum of the helicity amplitudes as given in Eq. (2.9) according to Eq. (2.13).

\section{Relative signs of the helicity amplitudes}

From the previous section we obtained the helicity amplitudes by taking the square root of the differential cross section. The relative signs of these amplitudes is still to be determined. These signs can be found using both parity and charge conjugation relations [15]. We shall firstly consider parity and use the symmetry properties of the $H_{\{\lambda\}}$ for reactions of the type

$$
A+B \rightarrow C+D
$$

where $\lambda_{A}, \lambda_{B}, \lambda_{C}$ and $\lambda_{D}$ are the helicities of the particles in the above reaction and $S_{A}, S_{B}, S_{C}$ and $S_{D}$ are the spins of the particles. Let $\eta_{j}$ be the intrinsic parity of particle $\mathrm{j}$. Then we have the condition

$$
H_{-\lambda_{C}-\lambda_{D} ;-\lambda_{A}-\lambda_{B}}(\theta)=\eta(-1)^{\lambda-\mu} H_{\lambda_{C} \lambda_{D} ; \lambda_{A} \lambda_{B}}(\theta)
$$

where

$$
\begin{aligned}
\eta & =\frac{\eta_{C} \eta_{D}}{\eta_{A} \eta_{B}}(-1)^{S_{A}+S_{B}-S_{C}-S_{D}} \\
\lambda & =\lambda_{A}-\lambda_{B} \\
\mu & =\lambda_{C}-\lambda_{D} .
\end{aligned}
$$

Then using charge conjugation for the reaction

$$
A+\bar{A} \rightarrow D+\bar{D}
$$

we have

$$
H_{d \bar{d} ; a \bar{a}}(\theta)=(1)^{\lambda-\mu} H_{\bar{d} d ; \bar{a} a}(\theta) .
$$

We may use these two discrete symmetry properties to determine some of the relative signs of the helicity amplitudes. 
In [16] the reaction $e^{-} e^{+} \rightarrow \mu^{-} \mu^{+}$is discussed, and the overall sign of an amplitude is presented there. We may use this to infer the following signs for $\phi_{3}$ and $\phi_{4}$

$$
\begin{aligned}
& H(+-;+-)=H(-+;-+)=-\frac{1}{2}(1+\cos \theta) G_{M} \\
& H(+-;-+)=H(-+;+-)=-\frac{1}{2}(1-\cos \theta) G_{M} .
\end{aligned}
$$

The other helicity amplitudes in [16] are zero as the mass of the lepton is neglected there. The five independent amplitudes suggest an alternative labelling of helicity amplitudes in the $s$ channel compared with the $t$ channel. In what follows in this paper we have assigned the labels $\phi_{1-6}$ to the following amplitudes in the annihilation reaction $p \bar{p} \rightarrow l^{+} l^{-}$

$$
\begin{aligned}
& \phi_{1}^{(s)}=H(++;++)=H(--;--) \\
& \phi_{2}^{(s)}=H(++;--)=H(--;++) \\
& \phi_{3}^{(s)}=H(+-;+-)=H(-+;-+) \\
& \phi_{4}^{(s)}=H(+-;-+)=H(-+;+-) \\
& \phi_{5}^{(s)}=H(++;+-)=-H(++;-+)=-H(--;-+)=H(--;+-) \\
& \phi_{6}^{(s)}=H(+-;--)=-H(-+;--)=-H(-+;++)=H(+-;++) .
\end{aligned}
$$

Here $\phi_{1}^{(s)}=\phi_{2}^{(s)}$, which is not the case in the $t$ channel. We find that the definition of the amplitudes $\phi_{3}$ and $\phi_{4}$ is the same in both channels. This re-labelling of some of the helicity amplitudes in the case of $\phi_{5}$ and $\phi_{6}$ may be explained by the fact that the helicity does not change sign in the analytic continuation. For example the amplitude $\phi_{5}^{(t)}=H(+-;++)$ in the $t$ channel becomes $\phi_{5}^{(s)}=H(++;-+)$ in the $s$ channel. This fact was first observed in [13].

\section{$4 \quad$ Helicity amplitudes for $N \bar{N} \rightarrow \bar{p} p$}

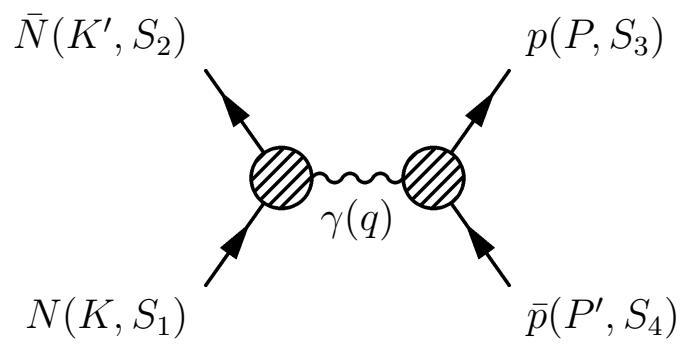

Figure 2: One photon exchange for $N+\bar{N} \rightarrow \bar{p}+p$ in the $s$ channel.

The next $s$ channel reaction we consider is the $N+\bar{N} \rightarrow \bar{p}+p$ annihilation, the time reverse of $\bar{p}+p \rightarrow N+\bar{N}$, figure 2. We can use the same momentum and 
spin variables as in Eq. (B.1) and Eq. (B.3) Using an alternative method to that used in section 2 it is possible to calculate the helicity amplitudes using the explicit form of the spinors. After Gordon decomposition the incoming nucleon current and outgoing proton current appear as

$$
u\left(K, S_{1}\right)\left[\gamma_{\mu} g_{M}+r_{\mu} f_{2}\right] \bar{v}\left(K^{\prime}, S_{2}\right) \bar{u}\left(P, S_{3}\right)\left[\gamma^{\mu} G_{M}+R^{\mu} F_{2}\right] v\left(P^{\prime}, S_{4}\right)
$$

where $f_{1}$ and $f_{2}$ are the form factors of the incoming structured particle $N$ of mass $m$ and $g_{M}=f_{1}+f_{2}$. In the above expression $F_{2}$ and $G_{M}=F_{1}+F_{2}$ refer to the proton form factors. The variables $R^{\nu}=\left(P^{\prime \nu}-P^{\nu}\right) / 2 M$ and $r_{\mu}=\left(K_{\mu}^{\prime}-K_{\mu}\right) / 2 m$.

Using the Dirac-Pauli representation the explicit form for the incoming spinors are

$$
u(K)=\left(\begin{array}{c}
E+m \\
2 k \lambda
\end{array}\right) \Psi_{\lambda}, \quad v\left(K^{\prime}\right)=\left(\begin{array}{c}
-2 k \lambda \\
E+m
\end{array}\right) \Psi_{\lambda}
$$

where $k$ is the magnitude of the centre of mass three momentum of the incoming particles. The matrix $\Psi_{\lambda}$ depends on the helicity, $\lambda$, and is given by

$$
\Psi_{+}=\left(\begin{array}{l}
1 \\
0
\end{array}\right), \quad \Psi_{-}=\left(\begin{array}{l}
0 \\
1
\end{array}\right) .
$$

In this representation the spinors of the final particles are

$$
v\left(P^{\prime}\right)=\left(\begin{array}{c}
-2 p \lambda \\
E+M
\end{array}\right) \psi_{\lambda}, \quad u(P)=\left(\begin{array}{c}
E+M \\
2 p \lambda
\end{array}\right) \psi_{\lambda}
$$

with

$$
\psi_{+}=\left(\begin{array}{r}
\cos \theta / 2 \\
\sin \theta / 2
\end{array}\right), \quad \psi_{-}=\left(\begin{array}{r}
-\sin \theta / 2 \\
\cos \theta / 2
\end{array}\right)
$$

where $E=\sqrt{s} / 2, \theta$ is the centre of mass scattering angle and $M$ is the mass of the proton.

Using this approach we find the following helicity amplitudes for the $N \bar{N} \rightarrow p \bar{p}$ process or its time reverse $p \bar{p} \rightarrow N \bar{N}$,

$$
\begin{aligned}
& H(++;++)=-\frac{2 \alpha m M}{s} \cos \theta G_{E} g_{E} \\
& H(+-;+-)=-\frac{\alpha}{2}(1+\cos \theta) G_{M} g_{M} \\
& H(+-;-+)=-\frac{\alpha}{2}(1-\cos \theta) G_{M} g_{M} \\
& H(++;+-)=-\frac{\alpha M}{\sqrt{s}} \sin \theta G_{E} g_{M} \\
& H(+-;++)=\frac{\alpha m}{\sqrt{s}} \sin \theta G_{M} g_{E}
\end{aligned}
$$


where $g_{M}=f_{1}+f_{2}$ and $g_{E}=f_{1}+f_{2} s / 4 M^{2}$ for the spinor particle $N$.

If $m=M$ in the above expressions then these are the helicity amplitudes for the annihilation reaction $p \bar{p} \rightarrow p \bar{p}$ with the familiar relation $\phi_{5}=-\phi_{6}$ for identical particle scattering. This relation is not true for the $s$ channel reaction $p \bar{p} \rightarrow l^{+} l^{-}$, Eq. (2.9). The above equations are consistent with the previous equations given in Section 3 for $p \bar{p} \rightarrow l^{+} l^{-}$when the form factors $f_{1} \rightarrow 1$ and $f_{2} \rightarrow 0$.

The spin averaged differential cross section for the process $N \bar{N} \rightarrow p \bar{p}$ is reproduced by the helicity amplitudes that we found.

$$
\begin{aligned}
\frac{d \sigma}{d \Omega}=\frac{\alpha^{2} \beta}{8 s} & \left\{\frac{8 m^{2}}{s}\left(\frac{4 M^{2}}{s} \cos ^{2} \theta\left|G_{E}\right|^{2}+\sin ^{2} \theta\left|G_{M}\right|^{2}\right)\left|g_{E}\right|^{2}\right. \\
+ & \left.2\left(\frac{4 M^{2}}{s} \sin ^{2} \theta\left|G_{E}\right|^{2}+\left(1+\cos ^{2} \theta\right)\left|G_{M}\right|^{2}\right)\left|g_{M}\right|^{2}\right\} .
\end{aligned}
$$

This is the sum of the five independent helicity amplitude found above as

$$
\begin{aligned}
\frac{d \sigma}{d \Omega} & =|H(++;++)|^{2}+|H(++;+-)|^{2}+|H(+-;++)|^{2} \\
& +\frac{1}{2}|H(+-;+-)|^{2}+\frac{1}{2}|H(+-;-+)|^{2} .
\end{aligned}
$$

This result also confirms the relationship between the 16 helicity amplitudes for $N \bar{N} \rightarrow p \bar{p}$ is the same as Eq. (2.13) for $p \bar{p} \rightarrow l^{+} l^{-}$. Using a linear combination of these helicity amplitudes according to [15] it is possible to reproduce the known expression for the single spin observable $A_{N}[5]$.

\section{Crossing relations for the helicity amplitudes}

We consider the process $A+B \rightarrow C+D$ ( $t$ channel). The crossed channel is then $B+\bar{D} \rightarrow C+\bar{A}$ ( $s$ channel) where the antiparticle of $A$ is denoted $\bar{A}$. The crossing relation between the two channels helicity amplitudes is then given by $[13,14]$

$$
\begin{aligned}
& H_{\lambda_{C} \lambda_{D} ; \lambda_{A} \lambda_{B}}^{(t)}(s, t)= \\
& \quad \sum_{\mu_{\bar{A}}, \mu_{B}, \mu_{C}, \mu_{\bar{D}}} d_{\mu_{\bar{A}} \lambda_{A}}^{t_{A}}\left(\omega_{A}\right) d_{\mu_{B} \lambda_{B}}^{t_{B}}\left(\omega_{B}\right) d_{\mu_{C} \lambda_{C}}^{t_{C}}\left(\omega_{C}\right) d_{\mu_{\bar{D}} \lambda_{D}}^{t_{D}}\left(\omega_{D}\right) H_{\mu_{C} \mu_{\bar{A}} ; \mu_{\bar{D}} \mu_{B}}^{(s)}(s, t)
\end{aligned}
$$

where $H_{\lambda_{C} \lambda_{D} ; \lambda_{A} \lambda_{B}}^{(t)}$ is the helicity amplitude in the $t$ channel and $\lambda_{i}$ is the helicity

of particle $i$. Likewise the helicity amplitude in the $s$ channel is $H_{\mu_{C}}^{(s)} \mu_{\bar{A}} ; \mu_{\bar{D}} \mu_{B}$ and the helicities of the particles are given as $\mu_{i}$.

The rotation functions $d_{\mu \lambda}^{j}$ for spinor particles are

$$
j=\frac{1}{2}: \quad d_{\frac{1}{2} \frac{1}{2}}(\beta)=\cos \frac{1}{2} \beta, \quad d_{-\frac{1}{2} \frac{1}{2}}(\beta)=\sin \frac{1}{2} \beta .
$$




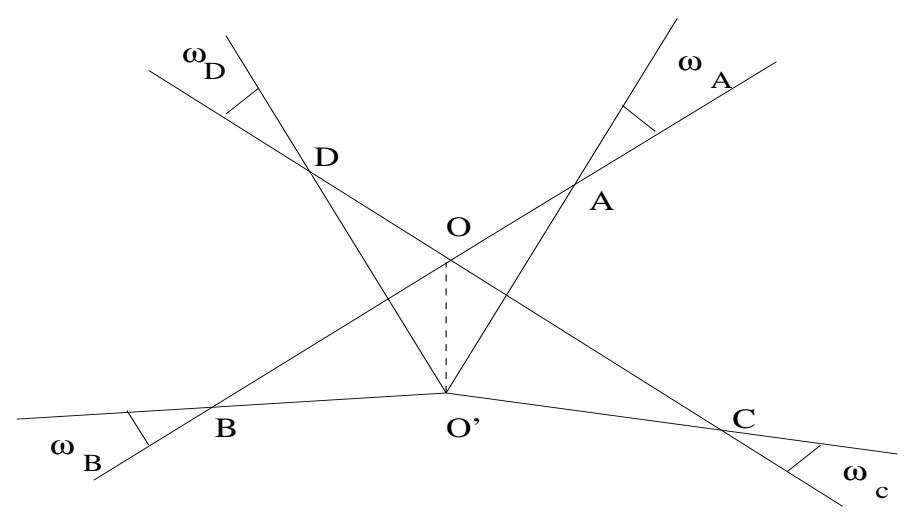

Figure 3: The velocity diagram for the transformation in Eq. (5.1) ; $O$ is the c.m. in the $t$ channel; $O^{\prime}$ is the c.m. in the $s$ channel.

Helicity amplitudes are generally given in a reference frame as they are not invariant under Lorentz transformations. The crossing relation for helicity amplitudes between the $s$ and the $t$ channel is obtained by first analytically continuing the $s$ channel centre of mass amplitude from the $s$ to the $t$ physical region and then performing a Lorentz transformation from the $s$ centre of mass frame to the $t$ centre of mass frame. The Wigner rotations, $\omega_{i}$, for this transformation are shown in figure 3 and are given by

$$
\cos \omega_{A}=\frac{-\left(s+m_{A}^{2}-m_{B}^{2}\right)\left(t+m_{A}^{2}-m_{C}^{2}\right)-2 m_{A}^{2} \Delta}{\mathcal{S}_{A B} \mathcal{T}_{A C}}
$$

where

$$
\begin{aligned}
\mathcal{S}_{i j}^{2} & =\left[s-\left(m_{i}+m_{j}\right)^{2}\right]\left[s-\left(m_{i}-m_{j}\right)^{2}\right] \\
\mathcal{T}_{i j}^{2} & =\left[t-\left(m_{i}+m_{j}\right)^{2}\right]\left[t-\left(m_{i}-m_{j}\right)^{2}\right] \\
\Delta & =m_{B}^{2}-m_{D}^{2}-m_{A}^{2}+m_{C}^{2} .
\end{aligned}
$$

Similarly the other rotation angles are given by

$$
\begin{aligned}
\cos \omega_{B} & =\frac{\left(s+m_{B}^{2}-m_{A}^{2}\right)\left(t+m_{B}^{2}-m_{D}^{2}\right)-2 m_{B}^{2} \Delta}{\mathcal{S}_{A B} \mathcal{T}_{B D}} \\
\cos \omega_{C} & =\frac{\left(s+m_{C}^{2}-m_{D}^{2}\right)\left(t+m_{C}^{2}-m_{A}^{2}\right)-2 m_{C}^{2} \Delta}{\mathcal{S}_{C D} \mathcal{T}_{A C}} \\
\cos \omega_{D} & =\frac{-\left(s+m_{D}^{2}-m_{C}^{2}\right)\left(t+m_{D}^{2}-m_{B}^{2}\right)-2 m_{D}^{2} \Delta}{\mathcal{S}_{C D} \mathcal{T}_{B D}} .
\end{aligned}
$$




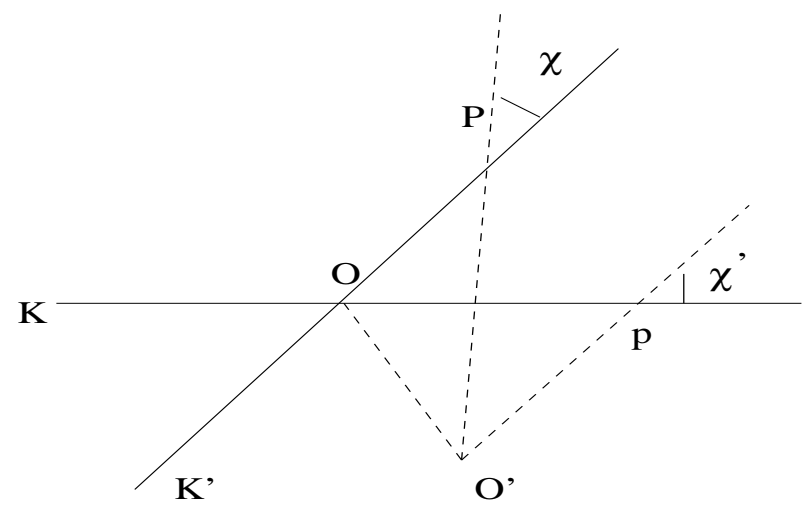

Figure 4: Velocity space diagram for initial $t$ channel scattering; $O$ is the c.m. in the $t$ channel; $O^{\prime}$ is the c.m. for the final configuration in the $s$ channel.

Using Eq.(5.1) we can the find the crossing matrix between the $t$ and $s$ channels. For lepton proton scattering $p l^{-} \rightarrow p l^{-}(t$ channel $)$ there are six independent helicity amplitudes while for proton antiproton annihilation, $p \bar{p} \rightarrow l^{+} l^{-}(s$ channel $)$ there are five independent helicity amplitudes. We may write the crossing relation as

$$
\phi_{i}^{(t)}=\sum_{j=1}^{6} W_{i j} \phi_{j}^{(s)}
$$

where $\phi_{1}^{s}=\phi_{2}^{s}$ in the $s$ channel.

For the previous $t$ and $s$ channel reactions we shall use the notation of [13] to present the crossing matrix, $W$, as

$W=\frac{1}{2}\left(\begin{array}{cccccc}\sin \psi \sin \chi & \sin \psi \sin \chi & 1-\cos \psi \cos \chi & 1+\cos \psi \cos \chi & -2 \sin \psi \cos \chi & 2 \cos \psi \sin \chi \\ 1-\cos \psi \cos \chi & -1-\cos \psi \cos \chi & \sin \psi \sin \chi & -\sin \psi \sin \chi & -2 \cos \psi \sin \chi & 2 \sin \psi \cos \chi \\ \sin \psi \sin \chi & \sin \psi \sin \chi & -1-\cos \psi \cos \chi & -1+\cos \psi \cos \chi & -2 \sin \psi \cos \chi & 2 \cos \psi \sin \chi \\ 1+\cos \psi \cos \chi & -1+\cos \psi \cos \chi & -\sin \psi \sin \chi & \sin \psi \sin \chi & 2 \cos \psi \sin \chi & -2 \sin \psi \cos \chi \\ -\sin \psi \cos \chi & -\sin \psi \cos \chi & -\cos \psi \sin \chi & \cos \psi \sin \chi & -2 \sin \psi \sin \chi & -2 \cos \psi \cos \chi \\ -\cos \psi \sin \chi & -\cos \psi \sin \chi & -\sin \psi \cos \chi & \sin \psi \cos \chi & 2 \cos \psi \cos \chi & 2 \sin \psi \sin \chi\end{array}\right)$

where the matrix entries are given in terms of Mandlestam variables as

$$
\begin{aligned}
& \cos \chi=-\cos \chi^{\prime}=\frac{\left(s+M^{2}-m^{2}\right) t}{\sqrt{t\left(t-4 M^{2}\right)\left[s-(M+m)^{2}\right]\left[s-(M-m)^{2}\right]}} \\
& \cos \psi=-\cos \psi^{\prime}=\frac{\left(s+m^{2}-M^{2}\right) t}{\sqrt{t\left(t-4 m^{2}\right)\left[s-(M+m)^{2}\right]\left[s-(M-m)^{2}\right]}} .
\end{aligned}
$$


Here $m$ and $M$ are the masses of the lepton and proton respectively. These are just the general Wigner rotations given before but for the specfic reactions $p \bar{p} \rightarrow l^{+} l^{-}$ and $N \bar{N} \rightarrow p \bar{p}$. When $M=m$ in Eq. (5.5) the matrix reduces to the already known result for $W$ [14]. The inverse of this crossing matrix is also discussed in [17] as a means of accessing the helicity amplitudes for $p \bar{p}$ scattering using the amplitudes for the $p p$ reaction.

The angle $\chi$ is shown in figure 5. In this diagram the momentum of the incoming particles in the $s$ channel is $K$ and $K^{\prime}$ while the outgoing momenta are $P$ and $P^{\prime}$. In the $t$ channel the incoming momenta are simply $P$ and $K$ while the outgoing momenta are $p=-P^{\prime}$ and $k=-K^{\prime}$. The point $O$ in figure 5 represents the centre of mass frame of $P$ and $p$ while $O^{\prime}$ is the centre of mass of $P$ and $K$.

As the helicity amplitudes for proton scattering in the $t$ channel are known [18] we may use this matrix, Eq. (5.5) in order to verify the signs for the crossed channel amplitudes Eq. (4.6) given in previous sections. We have found that using the above matrix Eq. (5.5) and the helicity amplitudes given in Eq. (2.9) and Eq. (4.6) we arrive at the correct $t$ channel amplitudes. We note here that using the crossing matrix and the expressions for the helicity amplitudes in the $s$ channel may be used as an alternative route in accessing the equivalent expressions in the $t$ channel $[18,19]$.

\section{Conclusions}

Proposed measurements of the electromagnetic form factors at PAX focus on annihilation reactions in the time like region. Detailed knowledge of these form factors is essential in order to test our current understanding of pQCD asymptotics in the time like region and dispersion theory. Recent experiments have raised serious issues concerning the electromagnetic form factors of hadrons. It is thought that further measurements in the time like region will shed light on these problems and allow us to improve the current form factor models.

Helicity transition amplitudes for proton antiproton electromagnetic reactions have been presented in this paper in the one photon approximation. These amplitudes contribute to the spin dependent observables for the elastic reaction in addition to hadronic amplitudes. We presented the helicity amplitudes for the reaction $p \bar{p} \rightarrow l^{+} l^{-}$including the lepton mass. As expected we found five independent amplitudes for this reaction. These results will be useful when considering mu or tau lepton pairs whose mass cannot be neglected. Expressions for the unequal mass reaction $N \bar{N} \rightarrow p \bar{p}$ were given in terms of four form factors. We found simple expressions for the five independent amplitudes which were found to sum to the spin averaged differential cross section for the reaction.

The helicity amplitudes in the time like region are simply related by analytic continuation to those in the space like region. We discussed the rotations needed to connect these two regions as well as presenting the $(6 \times 6)$ crossing matrix between the $s$ and $t$ channel. This matrix together with the helicity amplitude formulae given in this paper provide another way to compute the electromagnetic helicity amplitudes for the $e p \rightarrow e p$ and, more generally, the $p N \rightarrow p N$ elastic scattering reaction. 


\section{Acknowledgments}

EJ would like to thank the Irish Research Council for Science, Engineering and Technology (IRCSET) for a postgraduate research studentship and Trinity College Dublin for the award of a Scholarship. NHB is grateful to Enterprise Ireland for International Collaboration Programme grants that partially funded visits to Brookhaven National Laboratory at Upton, New York and to INFN at the University of Torino, Italy.

\section{A Appendix A}

The unpolarised differential cross section for s channel annihilation of spin half particles in the centre of mass system is

$$
\frac{d \sigma}{d \Omega}=\frac{\beta}{64 \pi^{2} s} \frac{1}{4} \sum_{\text {spin }}|\mathcal{M}|^{2}
$$

where $\mathcal{M}$ is the invariant amplitude for the process and $\beta$ is a flux factor. The Mandlestam variable $s$ is given in terms of the proton and antiproton momenta by

$$
s=\left(P_{\mu}+P_{\mu}^{\prime}\right)^{2}
$$

In an annihilation reaction of two spinor particles of mass $m_{i}$ producing a pair of mass $m_{f}$ the flux factor $\beta$ is given by

$$
\beta=\left(\frac{s-4 m_{f}^{2}}{s-4 m_{i}^{2}}\right)^{1 / 2} .
$$

The Dirac and Pauli form factors, $F_{1}$ and $F_{2}$, are functions of $q^{2}$ and normalised at the origin such that $F_{1}(0)=1$ and $F_{2}(0)=\mu_{p}-1$ where $\mu_{p}$ is the magnetic moment of the proton. The Sachs electric and magnetic form factors are given by [20]

$$
\begin{aligned}
G_{E}(s) & =F_{1}(s)+\frac{s}{4 M^{2}} F_{2}(s) \\
G_{M}(s) & =F_{1}(s)+F_{2}(s) .
\end{aligned}
$$

By definition these form factors are equal at threshold $\left(s=4 M^{2}\right)$. In the Breit frame, $G_{E}$ and $G_{M}$ may be interpreted as the Fourier transforms of the charge and magnetisation distributions, respectively. 


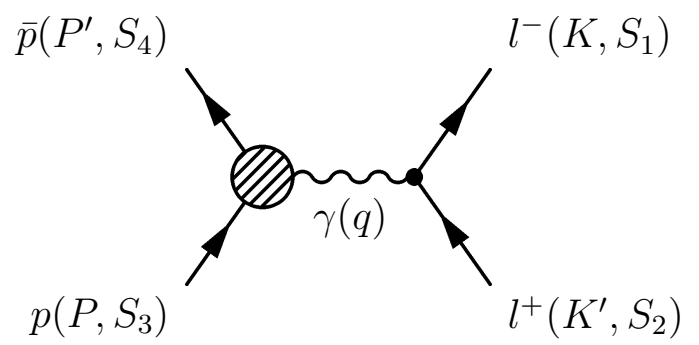

Figure 5: One photon exchange for $\bar{p}+p \rightarrow l^{-}+l^{+}$in the $s$ channel.

\section{B Appendix B}

For the reaction $\bar{p} p \rightarrow l^{+} l^{-}$we will be using the following momentum four vectors in the centre of mass system:

$$
\begin{aligned}
P^{\mu} & =(E, 0,0, p) \\
P^{\prime \mu} & =(E, 0,0,-p) \\
K^{\mu} & =(E, k \sin \theta, 0, k \cos \theta) \\
K^{\prime \mu} & =(E,-k \sin \theta, 0,-k \cos \theta)
\end{aligned}
$$

where the centre of mass scattering angle $\theta$ is given by

$$
\cos \theta=\frac{t-u}{\sqrt{s-4 m^{2}} \sqrt{s-4 M^{2}}}
$$

In order to include the spin-up and spin-down vectors in the following calculation we introduce the variables $\epsilon_{1}, \epsilon_{2}, \epsilon_{3}, \epsilon_{4}$ which are either \pm 1 depending on whether the spin is up or down.

$$
\begin{aligned}
S_{1} & =\frac{\epsilon_{3}}{m}(k, E \sin \theta, 0, E \cos \theta) \\
S_{2} & =\frac{\epsilon_{4}}{m}(k,-E \sin \theta, 0,-E \cos \theta) \\
S_{3} & =\frac{\epsilon_{1}}{M}(p, 0,0, E) \\
S_{4} & =\frac{\epsilon_{2}}{M}(p, 0,0,-E) .
\end{aligned}
$$

These spin four vectors are normalised to -1 and are perpendicular to the corresponding momentum four vector, e.g. $P_{\mu} S_{1}^{\mu}=0$. The momentum of the proton and the lepton are $p$ and $k$ respectively and are related to the energy, $E=\sqrt{s} / 2$, as

$$
\begin{aligned}
p & =\frac{1}{2} \sqrt{s-4 M^{2}} \\
k & =\frac{1}{2} \sqrt{s-4 m^{2}} .
\end{aligned}
$$




\section{References}

[1] M. Andreotti et al., Phys. Lett. B 559 (2003) 20.

[2] Jefferson Lab Hall A Collaboration, M. K. Jones et al., Phys. Rev. Lett. 84, 1398 (2000); Jefferson Lab Hall A Collaboration, O. Gayou et al., ibid. 88, 092301 (2001).

[3] V. Punjabi et al., Phys. Rev. C 71 (2005) 055202. [Erratum-ibid. C 71 (2005) 069902] arXiv:nucl-ex/0501018.

[4] F. Ambrosino et al., arXiv:hep-ex/0603056.

[5] N. H. Buttimore and E. Jennings, Eur. Phys. J. A 31, 9 (2007) arXiv:hep-ph/0607227.

[6] A. Bianconi, B. Pasquini and M. Radici, Phys. Rev. D 74 (2006) 034009 |arXiv:hep-ph/0606299]; A. Bianconi, B. Pasquini and M. Radici, Phys. Rev. D 74 (2006) 074012 [arXiv:hep-ph/0607277].

[7] V. Barone et al. [PAX Collaboration], arXiv:hep-ex/0505054.

[8] G. Bunce, N. Saito, J. Soffer, and W. Vogelsang, Annu. Rev. Nucl. Part. Sci. $50(2000) 525$.

[9] N.H. Buttimore, B.Z. Kopeliovich, E. Leader, J. Soffer, and T.L. Trueman, Phys. Rev. D 59,(1999) 114010.

[10] E. Tomasi-Gustafsson, F. Lacroix, C. Duterte and G. I. Gakh, Eur. Phys. J. A 24 (2005) 419. arXiv:nucl-th/0503001.

[11] A. Z. Dubničková, S. Dubnička and M. P. Rekalo, Nuovo Cim. A 109 (1996) 241; A. I. Akhiezer and M. P. Rekalo, Sov. Phys. Dokl. 13 (1968) 572.

[12] M. Jacob and G. C. Wick, Ann. Phys. (N. Y.) 7, (1959) 404.

[13] T. L. Trueman and G. C. Wick, Ann. Phys. (N. Y.) 26, (1964) 322.

[14] A. D. Martin and T. D. Spearman, Elementary Particle Theory, section 7.2.3, North-Holland Publishing Company, Amsterdam, 1970.

[15] E. Leader, Spin in Particle Physics, Cambridge University Press, Cambridge, 2001.

[16] Michael E. Peskin and Daniel V. Schroeder, An Introduction to Quantum Field Theory, section 1.7, Westview Press, New York, 1995.

[17] V. A. Okorokov and S. B. Nurushev, arXiv:hep-ph/0701226.

[18] D. S. O'Brien and N. H. Buttimore, arXiv:hep-ph/0609233.

[19] N. H. Buttimore, E. Gotsman, and E. Leader, Phys. Rev. D 18, (1978) 694.

[20] R. G. Sachs, Phys. Rev. 126 (1962) 2256. 